\title{
The LE cell: crime scene or crime stopper?
}

\author{
David S Pisetsky*
}

I have often said that the lupus erythematosus (LE) cell is the most important cell in all of medicine. This judgment does not reflect the cell's putative function in autoimmunity but rather the insights it has provided into the mechanisms of autoimmunity. Discovered fortuitously by Hargraves and colleagues (see Suggested reading below) at the Mayo Clinic, the test to detect this cell is very simple. A sample of peripheral blood is disrupted, incubated, and then spread onto a slide for inspection, after Wright's staining, under an old-fashioned light microscope. If the patient has lupus, then, voilà, the LE cell stands out among the mundane and ordinary red and white cells that populate the blood.

No doubt, my view of the significance of the LE cell is parochial and relates to my identity as a rheumatologist who has spent a career trying to solve the enigmas of anti-DNA antibodies. The competition for the LE cell is also not that stiff. 'Tart' cells are now forgotten and the Reed-Sternberg cell, though valuable for the diagnosis of Hodgkin's disease, has a very confusing phenotype, representing a B cell of some kind. Truly, what has the Reed-Sternberg cell revealed about the basis of malignancy? In contrast, the study of the LE cell has been a bonanza for autoimmunity.

As is now recognized, the LE cell phenomenon involves, in the presence of anti-nuclear antibodies (ANAs) and complement, the phagocytosis of a cell nucleus. In peripheral blood, the cell doing the engulfing is likely a neutrophil. For many years, the focus on the LE cell phenomenon was the ANA component and specificities that can bind nuclear molecules; this focus spawned simpler and more widely used tests such as the fluorescence anti-nuclear antibody (FANA) test. Other elements of the LE cell phenomenon raise salient issues that remain prime topics for investigation: the role of complement in the binding and clearance of nuclear material; the activation of neutrophils by nuclear molecules; and, perhaps most important, the existence of nuclear material outside cells.

*Correspondence: piset001@mc.duke.edu

Duke University Medical Center, 151G, Durham VA Medical Center, Durham, NC 27705, USA
Of events key to the creation of an LE cell, the translocation of an intact nucleus from the inside to the outside of a cell is perhaps the most mysterious and, for many years, attracted little investigative interest. Physiological enucleation is thought to occur essentially only in the bone marrow during red cell development and in the eye during the formation of the lens. Otherwise, cells quite reasonably hold onto their nuclei with their treasures of genetic information. As suggested by the apparent supply of nuclei available for the LE cell phenomenon, enucleation of hematopoietic cells perhaps occurs readily in the blood, a feature of a cell death process yet to be described and defined. If such a process exists among blood cells, it differs from apoptosis, in which nuclei condense and fragment for safe disposal in the form of apoptotic bodies and microparticles.

The enucleation central to the LE phenomenon also must differ from the extrusion of nuclear material from neutrophils as neutrophil extracellular traps (NETs). NETs are a unique form of DNA designed to entrap and ensnare bacteria and fungi. The process of the formation of NETs is called NETosis. During NETosis, which can be triggered by a variety of stimuli (including cytokines and Toll-like receptor (TLR) ligands), the nuclear membrane breaks down to allow mixing of DNA with cytoplasmic and granule contents which emerge from cells as strands or mesh. A NET should differ in appearance from the seemingly intact nucleus inside the LE cell. The striking images of NETs by fluorescence microscopy may not be the true story, however. Perhaps, during NETosis, the neutrophil ejects a more intact structure that unravels and disassembles over time once outside the cell to produce the now-classic image of a NET.

Whatever the mechanism for the generation of an LE cell, its close association with lupus, especially the more severe manifestations, has enforced the idea that it represents a pathological process linked to inflammation. Furthermore, the demonstration of LE cells in pleural effusions, for example, places the LE cell directly at the site of pathology. Even if the LE cell is an in vitro finding, the evidence for its involvement in pathogenicity, though circumstantial, is compelling. In other words, the LE cell has the tell-tale signs of a crime scene. All that is missing is the yellow tape the police use to keep gawkers away. 
I do not watch the CSI shows, nor am I an expert in forensics. But I am not ready to declare the case closed and the LE cell guilty as charged. I think that another interpretation is possible, and the LE cell as shown under the microscope signifies something quite different. Let me reconstruct the crime scene in a different way, which I hope will encourage a more general examination of the role of ANAs in lupus. This interpretation stems from exciting studies on the treatment of immune-mediated disease by monoclonal antibodies to nuclear molecules.

As shown now in many studies, the boundary between foreign (non-self) and self in the induction of innate immunity is not that sharp, and many self molecules can acquire immunological activity. This acquisition occurs during the exposure of these molecules to the immune system during cell injury and death, and the death processes often alter their structure by cleavage, denaturation, or post-translational modification. Such self molecules go by a number of terms; 'danger' molecules is the most dramatic and vivid description of their ability to signal that the organism is in dire straits. These molecules can also alert the immune system to trouble, hence the term alarmin. Finally, these molecules, by analogy to PAMPs, or pathogen-associated molecular patterns, can be called DAMPs, or damage-associated (or deathassociated) molecular patterns. Indeed, DAMPs and PAMPs can trigger the same set of sensors, such as the TLRs. The interaction of DAMPs and PAMPs with the same receptors indicates that the immune system does not really discriminate self from foreign or non-self but rather recognizes patterns that are structurally similar despite disparate origins.

Among the most prominent and potent DAMPs are nuclear molecules, and HMGB1 (high-mobility group box 1 protein) is at the top of the list. HMGB1 is a nonhistone nuclear protein that can bind DNA and serves as the prototype alarmin. HMGB1 leaves cells during cell death (apoptosis and necrosis), activation (especially of macrophages), and NETosis, and its extracellular activity is determined in part by its redox state. In addition, HMGB1 can partner with other molecules, such as lipopolysaccharide or cytokines like interleukin-1, to create new immunostimulatory moieties. Relevant to this discussion are observations that antibodies to HMGB1 are highly effective therapies in animal models of sepsis and arthritis. Coupled with the observations that HMGB1 is elevated in the blood or tissue during inflammatory disease, these findings have made HMGB1 a compelling target in new treatments.

Other nuclear components with immunological activity are nucleosomes, histones, and DNA, although DNA may need a partner like HMGB1 or LL37 for its immunostimulatory activity. Interestingly, histones can directly inflict tissue injury, and monoclonal anti-histone
(H1 and H3) antibodies are effective in blocking models of thrombosis, shock, and liver injury in mice. Together, these considerations suggest that nuclear material can be pathogenic and that ANAs (anti-HMGB1 and antihistones) can be therapeutic. Thus, the binding of an ANA can prevent disease as well as cause it.

In light of these data, the LE cell can be reconceptualized as a beneficial response, in which the binding of the nucleus facilitates neutralization or removal of a source of DAMPs. This process would be directly analogous to the opsonization and phagocytosis of a bacterium to remove a bundle of PAMPs before they cause harm, such as shock. Another way to look at this is that, if the target antigen in the LE cell were a bacterium instead of nucleus, you would say, 'Way to go, immune system. Get that critter.' Because the target is a nucleus, however, you say, 'Omigod, it's lupus. Get steroids'.

Although this line of thought sounds reasonable (at least to me), one can argue that there is an obvious flaw since the LE cell is associated with a more serious set of disease manifestations. If LE cells were preventing inflammation and damage, the opposite relationship could be expected. Thus, owing to a robust protective response, individuals with high numbers of LE cells (a sign of antinuclear defense) should display disease that is less severe, not more severe. I would counter this argument by saying that, in those individuals with active disease with LE cells, the system is working in overdrive but has been overwhelmed with nuclear material. This situation may be exacerbated in lupus by inadequate handling of the burden of dangerous molecules. Clearance defects abound in patients with lupus, providing plausibility for this model. In this scenario, the presence of LE cells at sites of inflammation does not mean that the cells are committing a crime but that they are trying to stop or prevent one.

A further issue in conceptualizing the LE cell relates to the heterogeneity in the serological response of patients with lupus. These responses are highly diverse, targeting a multitude of nuclear antigens. Importantly, many ANAs have never been definitively associated with clinical disease manifestations, and some patients can remain 'serologically positive, clinically negative' for many years, seemingly spared active disease despite abundant ANA production. In such patients, this discordancy suggests that the ANAs, though pathological, are neither pathogenic nor nephritogenic. Mouse transfer models show clearly that, although some anti-DNAs can deposit in the kidney, many cannot despite bona fide DNA binding. Clearly, interaction with a nuclear antigen does not necessitate a role in promoting disease, or exclude a role in preventing disease. It is of interest, therefore, that between $15 \%$ and $25 \%$ of otherwise healthy individuals can express an ANA when blood is assayed by sensitive 
techniques. A high frequency of ANA expression in normal individuals could signify a propensity to autoreactivity in the general population but could also indicate the ordinary operation of immunoregulatory mechanisms to constrain immune responses to nuclear molecules. Unfortunately, the specificity of the ANAs in normal individuals is usually not known, although the characterization of these ANAs could provide additional insights into the way that the immune system protects against the activity of nuclear molecules. Therefore, it is of great interest that, in a study by $\mathrm{Li}$ and colleagues (see Suggested reading below), healthy controls without ANA reactivity showed more evidence of upregulated gene expression by array analysis of peripheral blood cells than healthy controls with ANA positivity; those with ANA positivity, however, showed evidence of upregulation of genes associated with the interferon signature. Therefore, further genetic and genomic studies of patients and controls should be informative in elucidating immune disturbances underlying lupus, especially if studies allow for the possibilities that ANAs can act defensively as well as offensively and that serological disturbances can indicate a response to impending danger and not necessarily its cause.

The remarkable success of anti-HMGB1 antibodies in animal models of disease and the equally impressive but more limited studies on anti-histone antibodies indicate that some ANAs are protective. This protection could result from increased clearance of nuclear antigens or blockade of TLR binding. Since many nuclear molecules with DAMP activity function in the context of a nanostructure or microstructure (for example, microparticles or microvesicles), protective ANAs could indeed promote their phagocystosis after opsonization, effectively reproducing the LE cell phenomenon. This discussion is not just for intellectual amusement but rather to suggest new lines of research into the serology of lupus and other diseases characterized by ANA production. Given the possibility that ANAs can be protective and not just pathogenic (or non-pathogenic), the relationship between serology and clinical disease events may be clarified. Furthermore, the possibility that some ANAs are protective could spur inquiry into new biological therapies based on the paradigm, now so well established with anti-HMGB1 antibodies, that ANAs can block disease by eliminating deleterious nuclear material. If the LE cell phenomenon provided evidence for the prevention of disease and not its induction, then a venerable but clunky assay could have a revival as a screen for agents to stop the immunological crime known as lupus.

\section{Abbreviations}

ANA, anti-nuclear antibody; DAMP, damage-associated (or death-associated) molecular pattern; HMGB1, high-mobility group box 1 protein; LE, lupus erythematosus; NET, neutrophil extracellular trap; PAMP, pathogen-associated molecular pattern; TLR, Toll-like receptor

Published: 26 June 2012

\section{Suggested reading}

Andersson U, Tracey KJ: HMGB1 is a therapeutic target for sterile inflammation and infection. Annu Rev Immunol 2011, 29:139-162.

Farrell K, Jarrett RF: The molecular pathogenesis of Hodgkin lymphoma. Histopathology 2011, 58:15-25

Griffith TS, Ferguson TA: Cell death in the maintenance and abrogation of tolerance: the five Ws of dying cells. Immunity 2011, 35:456-466.

Gulhane S, Gangane N: Detection of lupus erythematosus cells in pleural effusion: an unusual presentation of systemic lupus erythematosus. $\int$ Cytol 2012, 29:77-79.

Hargraves M, Richmond H, Morton R: Presentation of two bone marrow elements; the tart. Proc Staff Meet Mayo Clin 1948, 23:25-28.

Harris $\mathrm{HE}$, Andersson U, Pisetsky DS: HMGB1: a multifunctional alarmin driving autoimmune and inflammatory disease. Nat Rev Rheumato/ 2012, 8:195-202

Hebederen historical series: The LE cell. Rheumatology 2001, 40:826-827. Hreggvidsdottir HS, Lundberg AM, Aveberger AC, Klevenvall L, Andersson U, Harris HE: High mobility group box protein 1 (HMGB1)-partner molecule complexes enhance cytokine production by signaling through the partner molecule receptor. Mol Med 2012, 18:224-230.

Keerthivasan G, Wickrema A, Crispino JD: Erythroblast enucleation. Stem Cells Intl $2011,139851$.

Kruse K, Janko C, Urbonaviciute V, Mierke CT, Winkler TH, Voll RE, Schett G, Munoz $L E$, Hermann M: Inefficient clearance of dying cells in patients with SLE; anti-dsDNA autoantibodies, MFG-E8, HMGB-1 and other players. Apoptosis 2010, 15:1098-1113.

Li Q-Z, Zhou J, Lian Y, Zhang B, Branch VK, Carr-Johnson F, Karp DR, Mohan C, Wakeland EK, Olsen NJ: Interferon signature gene expression is correlated with autoantibody profiles in patients with incomplete lupus syndromes. Clin Exp Immunol 2009, 159:281-291.

Mortensen ES, Rekvig OP: Nephritogenic potential of anti-DNA antibodies against necrotic nucleosomes. J Am Soc Nephrol 2009, 20:696-704.

Nakano T, Goto S, Lai C-Y, Hsu L-W, Takaoka Y, Kawamoto S, Chiang K-C, Shimada Y, Ohmori N, Goto T, Sato S, Ono K, Cheng Y-F, Chen C-L: Immunological aspects and therapeutic significance of an autoantibody against histone $\mathrm{H} 1$ in a rat model of concanavalin A-induced hepatitis. Immunology 2010, 129:547-555.

Quan-Zhen L, Karp DR, Quan J, Branch VK, Zhou J, Lian Y, Chong BF, Wakeland EK, Olsen NJ: Risk factors for ANA positivity in healthy persons. Arthritis Res Ther 2011, 13:1-11.

Pisetsky D: Cell death in the pathogenesis of immune-mediated diseases: the role of HMGB1 and DAMP-PAMP complexes. Swiss Med Wkly 2011, 141:w13256.

Park JY, Malik A, Dumoff KL, Gupta PK: Case report and review of lupus erythematosus cells in cytology fluids. Cytopathol 2007, 35:806-809.

Ramijsen Q, Kuijpers TW, Wirawan E, Lippens S, vandenabeele P, Vanden-Berghe T: Dying for a cause: NETosis, mechanisms behind an antimicrobial cell death modality. Cell Death Differ 2011, 18:581-588.

Xu J, Zhang X, Pelayo R, Monestier M, Ammollo CT, Semeraro F, Taylor FB, Esmon NL, Lupu F, Esmon CT: Extracellular histones are major mediators of death in sepsis. Nature Med 2009, 15:1318-1322.

Xu J, Zhang X, Monestier M, Esmon NL, Esmon CT: Extracellular histones are mediators of death through TLR2 and TLR4 in mouse fatal liver injury. J Immuno/ 2011, 187:2626-2631.

doi:10.1186/ar3878

Cite this article as: Pisetsky DS: The LE cell: crime scene or crime stopper? Arthritis Research \& Therapy 2012, 14:120. 